\title{
Oxidant/antioxidant balance in patients with thyroid cancer ${ }^{1}$
}

\author{
Melih Akinci', Funda Kosova", Bahadir Çetin" ${ }^{\text {III }}$ Aylin Sepici" ${ }^{\mathrm{IV}}$, Nilgün Altanv, Sebahattin Aslan ${ }^{\mathrm{VI}}$, Abdullah Çetin ${ }^{\mathrm{VII}}$

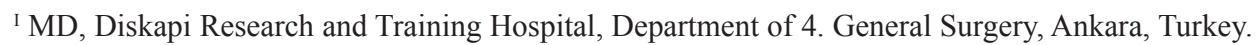 \\ ${ }^{\text {II }}$ MD,Celal Bayar University, Department of Biochemistry, Manisa, Turkey. \\ III MD, Associate Prof, Ankara Oncology Hospital, Department of 1. General Surgery, Ankara, Turkey. \\ Iv MD, Gazi University, School of Medicine, Department of Medical Biochemistry, Ankara, Turkey. \\ ${ }^{v}$ MD, Gazi University, School of Medicine, Department of Medical Biochemistry, Ankara, Turkey.

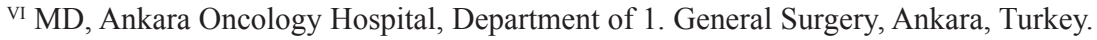 \\ ${ }^{\mathrm{VII}} \mathrm{MD}$, Ankara Oncology Hospital, Department of 1. General Surgery, Ankara, Turkey.
}

\begin{abstract}
Purpose: To compare the antioxidant enzyme activities of superoxide dismutase (SOD), glutathione peroxidase (GSH-Px) and the levels of lipid peroxidation product malondialdehyde (MDA) in blood samples of thyroid cancer patients compared to healthy controls. Methods: 43 control subjects (mean age $44 \pm 13$ years) and 43 patients ( $43 \pm 13$ years) presented with multinodular goiter whose fine needle aspiration revealed malignant cytology were included into this study. The SOD, MDA and GSH-Px activities were measured in control subjects, and before/20 days after thyroidectomy in thyroid cancer patients. Results: SOD activities of pre-thyroidectomy, post-thyroidectomy and control groups were not different $(\mathrm{p}>0.05)$. Before thyroidectomy GSH-Px activities were lower $(\mathrm{p}<0.05)$ and MDA levels were higher $(\mathrm{p}<0.05)$ than the control group. In post- thyroidectomy, GSH-Px activity $(\mathrm{p}<0.05)$ increased, and MDA levels $(\mathrm{p}<0.05)$ decreased compared to prethyroidectomy levels. After thyroidectomy GSH-Px activity was significantly higher than the control group $(\mathrm{p}<0.05)$. Although post-thyroidectomy MDA levels significantly decreased, they were still higher than the control group $(\mathrm{p}<0.05)$. Conclusion: The superoxide dismutase does not seem to change with thyroid cancer and thyroidectomy but both antioxidant glutathione peroxidase and lipid peroxidation product malondialdehyde do. These preliminary findings may point out oxidant/antioxidant imbalance associated with thyroid cancer.
\end{abstract}

Key words: Thyroid Neoplasms. Enzymes, Antioxidants. Malondialdehyde. Superoxide Dismutase. Glutathione Peroxidase.

${ }^{1}$ Ankara Oncology Hospital, Department of 1. General Surgery, Ankara, Turkey.

\section{Introduction}

Oxidative stress, which is characterized as a misbalance between free radicals and antioxidants in favour of radicals, participates in the pathogenesis of many diseases and their complications ${ }^{1}$. Reactive oxygen species (ROS) consisting mainly of superoxide, hydrogen peroxide and hydroxyl radical, have been conventionally considered to have carcinogenic potential ${ }^{2}$ and to promote invasiveness ${ }^{3}$.

The levels of ROS are controlled by antioxidant enzymes, such as superoxide dismutase (SOD), glutathione peroxidase (GSH-Px). Serum malondialdehyde (MDA) level as a marker for lipid peroxidation is commonly used as an indicator for oxidative damage in cells and tissues ${ }^{4}$. The balance between the formation and removal of lipid peroxides determines the peroxide level in cells. This balance can be disturbed if cellular defences are decreased or if there is a significant increase in peroxidative reactions ${ }^{5}$.

The oxidative stress is common in the thyroid tissue during utilization of $\mathrm{H}_{2} \mathrm{O}_{2}$ for thyroxin synthesis, the inflammation produces ROS, and when tumour has active proliferation ${ }^{6,7}$. However, very little is known about oxidant/antioxidant balance in thyroid cancer ${ }^{8}$. Yet, there is not any study evaluating the effect of complete resection of the thyroid tissue on this balance.
Thus, in order to further delineate the role of oxidant antioxidant balance in thyroid cancer, the aim of this study was to evaluate the antioxidant enzyme activities such as SOD, GSH-Px and the level of lipid peroxidation product MDA in blood samples of thyroid cancer patients before and after the thyroidectomy operation compared with healthy controls.

\section{Methods}

This is an age-and sex-matched case-control study, conducted at Department of Medical Biochemistry of Gazi University of Medicine and Department of General Surgery of Ankara Oncology Hospital, Ankara, Turkey. The study was approved by the hospital Ethical committee. All the patients and the volunteers involved in the study gave informed consent.

\section{Patients}

Recruitment of subjects was performed by convenience sampling at outpatient General Surgery Clinic of Oncology Hospital, Turkey by trained physicians. 45 patients and 43 control subjects were included in this study. The patients with female gender presented with multinodular goiter whose fine needle aspiration revealed malignant cytology of papillary carcinoma 
of the thyroid. As follicular carcinoma of the thyroid cannot be diagnosed by fine needle aspiration, only the patients with papillary morphology were included into the study. Total thyroidectomy was performed after which they came to a control visit on the postoperative twentieth day. Patients and control groups were euthyroid with normal thyroid function tests and had normal range of levels for total cholesterol and triglycerides. Patients and controls with Diabetes Mellitus, hypertension, on hormonal therapy such as oral contraceptives, thyroxin derivates, estrogens replacement therapy and also medications for chronic diseases were excluded. Two patient were excluded because of not performing total thyroidectomy so 43 patients were enrolled into the study group. The controls were age, sex matched healthy volunteers.

\section{Assay}

Blood samples were kept at $-70^{\circ} \mathrm{C}$ until analysis. All samples from each patient were run in the same assay.

Serum thyroid hormones were measured by radioimmunoassay kits (TOSOH). Total cholesterol and triglycerides were measured by commercially available enzymatic reagents adapted to auto analyzer.

For SOD assay, serum samples were chloroform and ethanol $(3: 5 \mathrm{v} / \mathrm{v})$ was added. This mixture was centrifuged at $5000 \mathrm{~g}$ for $2 \mathrm{hr}$. The supernatant was used for SOD assay, using xanthine oxidase as superoxide generator ${ }^{9}$. The results were expressed as unit per ml. One unit of SOD is defined as the amount of protein that inhibits the rate of nitro blue tetrazolium (NBT) reduction by $50 \%$.

GSH-Px activity was measured by the method of Paglia and Valentine and the activity was expressed as micromoles of NADPH oxidized to NADP per minute/ $\mathrm{ml}$ using an extinction coefficient for NADPH at $340 \mathrm{~nm}^{10}$.

MDA levels were measured as thiobarbituric acid reactive substances according to the modified method of Uchiyama at $532 \mathrm{~nm}^{11}$ and the results were obtained in $\mathrm{nmol} \mathrm{MDA} / \mathrm{ml}^{11}$.

\section{Statistical analysis}

Parametric methods were performed in the statistical analysis of biomedical data ( $\mathrm{t}$ test and paired $\mathrm{t}$ test). Two -tailed probability $(p)$ values were calculated throughout, and statistical significance was defined as $p<0.05$. All analyses were performed by statistical software SPSS 11.5.

\section{Results}

Age, sex (all of the subjects were female), weight and height were not significantly different between the thyroid cancer patients and the control group subjects (Table 1). There were more postmenopausal subjects in the thyroid cancer group compared to the control group $(\mathrm{p}<0.05)$ (Table 1$)$. The thyroid hormones, total cholesterol and triglycerides levels were within normal limits in all subjects.

TABLE 1 - Demographical variables

\begin{tabular}{l|c|c}
\hline & Control & Thyroid cancer patients \\
\hline Age (years) & $44 \pm 13$ & $43 \pm 13$ \\
\hline Weight (kg) & $71 \pm 14$ & $68 \pm 13$ \\
\hline Height (cm) & $163 \pm 9$ & $161 \pm 7$ \\
\hline Menopausal status (pre/post) & $38 / 5$ & $30 / 13 *$ \\
\hline
\end{tabular}

* $\mathrm{P}=0.03$

As we mentioned before two patients were excluded because of nonmalignant postoperative pathology. Remaining forty three patients had postoperative papillary carcinoma histology. Five of them had occult carcinoma (i.e. 1,0cm or less tumoral mass diameter without local invasion). Remaining thirty eight patients had tumours larger than $1,0 \mathrm{~cm}$ diameter. Four patients with more than $1,0 \mathrm{~cm}$ tumoral mass diameter had capsule and local structure invasion such as trachea, oesophagus and recurrent laryngeal nerve.

Superoxide dismutase (SOD), glutathione peroxidase (GSH-Px) and malondialdehyde (MDA) levels of before- thyroidectomy, post-thyroidectomy and control groups are shown Table 2. In the present study, before thyroidectomy, SOD activity was not significantly different $(\mathrm{p}>0.05)$ but GSH-Px activity was lower and MDA levels ( $\mathrm{p}<0.01$ ) was higher compared to controls. In post- thyroidectomy, GSH-Px activity increased significantly and MDA levels decreased $(\mathrm{p}<0.01)$ significantly but SOD activity did not change when compared to prethyroidectomy levels. Although post-thyroidectomy, increased GSH-Px levels were significantly higher than control group $(\mathrm{p}<0.05)$ and SOD activity was similar to the control group, decreased MDA $(\mathrm{p}<0.01)$ levels were still significantly higher than the control group. 
TABLE 2 - Antioxidants enzyme activities and MDA levels in thyroid cancer

\begin{tabular}{l|c|c|c}
\hline & SOD & GPx (u/mL) & MDA (nmol/mL) \\
\hline Control & $11,15+0,34$ & $173,98+4,86$ & $1,38+0,33$ \\
\hline & & & \\
Before thyroidectomy & $10,98+0,41$ & $162,76+11,82^{*}$ & $3,61+0,73^{*}$ \\
\hline & & & $2,96+0,84^{* \#}$ \\
Post- thyroidectomy & $10,20+0,59$ & $217,15+9,71^{* \#}$ & 2 \\
$\begin{array}{l}* \mathrm{P}<0.05 \text { compared to control } \\
\text { \# }<0.05 \text { compared to prethyroidectomy levels }\end{array}$
\end{tabular}

\section{Discussion}

According to our results, serum SOD does not seem to change with thyroid cancer and thyroidectomy but both antioxidant GSH-Px and lipid peroxidation product MDA do. These preliminary findings may point out oxidant/antioxidant imbalance associated with thyroid cancer.

In thyroid, reactive ROS and free radicals participate in physiological and pathological process in the gland. Much evidence gathered in the last years involves the free radicals in the mechanisms of initiation, development of neoplastic transformations in vivo and in vitro, as well as in the activity of specific oncogenes. Most of them come from the fact that the agents that remove the free radical or interfere in the chain of events induced by free radical can inhibit the neoplastic process both at cellular and molecular level ${ }^{8,12}$.

Free radicals affect the cell components such as lipid, protein, DNA and carbohydrates, of which lipids are the most sensitive part. Serum MDA level, as a marker for lipid peroxidation, is commonly used as an indicator for oxidative damage in cells and tissues. Hence, we determined the MDA levels as the stable end product of lipid peroxidation. In our study, before thyroidectomy and post-thyroidectomy, blood MDA levels were significantly higher, compared to controls. However, in post thyroidectomy, the serum MDA level significantly decreased compared to prethyroidectomy levels but was still significantly higher than the control group's level.

In fact serum enzyme activities or MDA levels are good indicators for the systemic oxidant/antioxidant status but not necessarily indicate their real changes occurring in the thyroid directly, because numerous factors are involved and can modify the serum outcome. However, we standardized our patients and control group in order to select patients and controls with unaffected hormone profile who had normal thyroid function tests and had normal levels of total cholesterol and triglycerides. Patients and controls with Diabetes Mellitus, hypertension or on hormonal therapy such as oral contraceptives, thyroxin derivates, estrogens replacement therapy and also medications for chronic diseases were excluded. Mano et al. ${ }^{4}$ and Sadani et al. ${ }^{8}$ showed that the lipid peroxide concentration, expressed as MDA concentration, was significantly higher in the specimens from papillary carcinoma than those in the normal thyroid tissue. The increase of free radicals in thyroid cancer condition is suggested to be due to the increased lipid peroxidation and the damage of antioxidant defence system ${ }^{13}$. In our study, in thyroid cancer patients MDA levels before thyroidectomy were found to be higher than those in agematched controls indicating increased free radical generation. It is obvious that there are clues, there is an oxidant shift in thyroid cancer patients with normal hormone profile. At least we think that MDA level may have a value for control visits of thyroid cancer patients after thyroidectomy and there is no doubt that more investigations are needed.

Sugawara et al. ${ }^{14}$ and Durak et al. ${ }^{15}$ showed that endemic goiter tissues contained significantly lower SOD activity and concentration compared with those of normal tissues. In various other thyroid disorders including thyroid cancer SOD also found to be low. However in our study, both pre and post operative SOD activity was slightly lower than the control but it was not statistically different. SOD activity seems to play insignificant role in thyroid cancer development in our series but it is not possible to reach a certain decision as the number of patients is low and this must be investigated in larger series.

Aerobic organisms have a defence mechanism to prevent oxidative damage to tissues and cells and include an array of antioxidant enzymes including glutathione peroxidase and glutathione reductase. The function of glutathione peroxidase is to catalyze the reduction of $\mathrm{H}_{2} \mathrm{O}_{2}$ to water as well as a number of hydroperoxides e.g., DNA and lipid peroxides, to alcohols by using reduced glutathione. Glutathione reductase reduces oxidized glutathione to reduced glutathione, by using NADPH as the electron source. 
GSH-Px is primarily responsible for decomposition of $\mathrm{H}_{2} \mathrm{O}_{2}$. In normal cells, the activity of antioxidant enzymes either increase or decrease, depending on the tissue and age of humans ${ }^{16,17}$. Durak et al. ${ }^{15}$ showed that GSH-Px activities were lower in carcinomas ${ }^{15}$. In the present study, before thyroidectomy, GSH-Px activity was lower in the thyroid cancer patients than the respective control group. However, after thyroidectomy, GSH-Px significantly increased compared to before -thyroidectomy.

\section{Conclusion}

In conclusion increased antioxidant GSH-Px activity and unchanged SOD activity together with decreased lipid peroxidation product MDA after thyroidectomy indicate that in thyroid cancer antioxidants enzyme activity are decreased and lipid peroxidation products are elevated. The free radical levels and the antioxidant activities do not return to normal levels 20 days after the resection of the thyroid gland. These levels may or may not change with longer duration of time. Given the lack of any other study examining the change in oxidant antioxidant balance in thyroid cancer after thyroidectomy, further studies are required to examine longer time intervals such as 3 or 6 months after the operation. These studies may have treatment implications such as antioxidant therapy or duration of any perioperative intervention. It would also be interesting to see the local levels in the extracted thyroid tissue and the effects of hormone replacement therapy on the oxidant /antioxidant balance in these patients.

\section{References}

1. Bankson DD, Kestin M, Rifai N. Role of free radicals in cancer and atherosclerosis. Clin Lab Med. 1993;13:463-80.

2. Ames BN. Measuring oxidative damage in humans: relation to cancer and aging. IARC Sci Publ. 1988;89:407-16.

3. Shinkai K, Mukai M, Akedo H. Superoxide radical potentiates invasive capacity of rat ascites hepatoma cell in vitro. Cancer Lett. 1986;1:7-13.
4. Mano T, Mano T, Shinohara R, Iwase K, Kotake M, Hamada M, Uchimuro K, Hayakawa N, Hayashi R, Nakai A, Ishizuki Y, Nagasaka A.Changes in free radical scavengers and lipid peroxide in thyroid glands of various thyroid disorders. Horm Metab Res. 1997;29:351-54.

5. Karbownik M, Lewnski A. The role of oxidative stress in physiological and pathological processes in the thyroid gland; possible involvement in pineal-thyroid interactions. Neuro Endocrinol Lett. 2003;24:293-303.

6. Yanagawa T, Ishikawa T, Ishii T, Tabuchi K. Peroxiredoxin I expression in human thyroid tumours. Cancer Lett. 1999;145:127-32.

7. Fujita T. Formation and removal of reactive oxygen species, lipid peroxides and free radicals, and their biological effects. Yakugaku Zasshi. 2002;122:203-18.

8. Sadani GR, Nadkarni GD. Role of tissue antioxidant defence in thyroid cancers. Cancer Lett. 1996;109:231-5.

9. Sun Y, Oberley LW, Li Y. A simple method for clinical assay of superoxide dismutase. Clin Chem. 1988;34:497-500.

10. Paglia DE, Valentine WN. Studies on the quantitative and qualitative characterization of erythrocyte glutathione peroxidase. J Lab Clin Med. 1967;70:158-69.

11. Uchiyama M, Mihara M. Determination of malonaldehyde precursor in tissues by thiobarbituric acid test. Ann Biochem. 1978;86:271-8.

12. Dumitrescu C, Belgun M, Olinescu R, Lianu L, Bartoc C. Effect of vitamin $\mathrm{C}$ administration on the ratio between the pro and antioxidative factors. Rom J Endocrinol. 1983;31:81-4.

13. Yanagawa T, Ishikawa T, Ishii T, Tabuchi K, Iwasa S, Bannai S, Omura $\mathrm{K}$, Suzuki H, Yoshida H. Peroxidoxin I expression in human thyroid tumours. Cancer Lett. 1999;145:127-32.

14. Sugawara M, Kita T, Lee ED, Takamatsu J, Hagen GA, Kuma K, Medeiros-Neto GA. Deficiency of superoxide dismutase in endemic goiter tissue. J Clin Endocr Metab. 1988;67:1156-61.

15. Durak I, Bayram F, Kavutcu M, Canbolat O, Oztürk HS. Impaired enzymatic antioxidant defence mechanism in cancerous human thyroid tissues. J Endocrinol Invest. 1996;19:312-5.

16. Danh HC, Benedetti MS, Dostert P. Differential changes in superoxide dismutase activity in brain and liver of old rats and mice. J Neurochem. 1983;40:1003-7.

17. Pinto RE, Bartley W. The effect of age and sex on glutathione reductase and glutathione peroxidase activities and on aerobic glutathione oxidation in rat liver homogenates. Biochem J. 1969;112:109-15.

Conflict of interest: none Financial source: none

\section{Correspondence:}

Melih Akinci

1468. sok. Lale apt. No 4/14

Cukurambar Ankara 06520

Phone: 00903122857997

Fax: 903123454979

melihakinci@yahoo.com

Received: June 18, 2008

Review: August 21, 2008

Accepted: September 23, 2008

\section{How to cite this article}

Akinci M, Kosova F, Çetin B, Sepici A, Altan N, Aslan S, Çetin A. Oxidant/antioxidant balance in patients with thyroid cancer. Acta Cir Bras. [serial on the Internet] 2008 Nov-Dec;23(6). Available from URL: $\underline{\text { http://www.scielo.br/acb }}$ 